\title{
O.S.P.
}

L'orientation scolaire et professionnelle

$47 / 1 \mid 2018$

L'héritage de Maurice Reuchlin

\section{WISC-V : une évolution attendue et nécessaire}

WISC-V: a waited and necessary evolution

Evelyne Laporte et Katia Terriot

\section{(2) OpenEdition}

Journals

Édition électronique

URL : http://journals.openedition.org/osp/5658

DOI : 10.4000/osp.5658

ISSN : 2104-3795

Éditeur

Institut national d'étude du travail et d'orientation professionnelle (INETOP)

Édition imprimée

Date de publication : 15 mars 2018

ISSN : 0249-6739

Référence électronique

Evelyne Laporte et Katia Terriot, «WISC-V : une évolution attendue et nécessaire », L'orientation scolaire et professionnelle [En ligne], 47/1 | 2018, mis en ligne le 15 mars 2020, consulté le 16 décembre 2020.

URL : http://journals.openedition.org/osp/5658 ; DOI : https://doi.org/10.4000/osp.5658

Ce document a été généré automatiquement le 16 décembre 2020.

(c) Tous droits réservés 


\title{
WISC-V : une évolution attendue et nécessaire
}

\author{
WISC-V: a waited and necessary evolution
}

Evelyne Laporte et Katia Terriot

\section{Introduction}

1 Les psychologues de l'Éducation nationale sont de plus en plus sollicité.e.s par les familles, les équipes éducatives, les commissions et les jeunes eux-mêmes pour apporter leur regard et leur expertise et les aider dans la compréhension de la situation, dans une prise de décision quant à une orientation, des préconisations, des aides pouvant être mises en place dans et hors de l'école, etc. Dans le cadre des diverses demandes dont il.elle.s peuvent être le.la dépositaire, certaines d'entre elles pourront nécessiter le recours à un bilan psychologique avec éventuellement l'utilisation d'une échelle de Wechsler comme le WISC si le.la praticien.ne le juge pertinent au regard de la demande, de la situation et des caractéristiques de la personne à évaluer. La dernière version française des échelles de Wechsler pour enfants et adolescent.e.s est parue en octobre 2016 et nécessite une appropriation par les psychologues.

Déjà, la nouvelle architecture introduite par la quatrième version des échelles de Wechsler (WISC-IV; 2005) a contraint le.la praticien.ne à changer de paradigme: partant d'une distinction empirique entre deux échelles (verbale et de performance), l'interprétation reposait dorénavant sur l'analyse d'indices dans un cadre théorique, en lien avec les facteurs de groupe de la théorie de Catell-Horn-Carroll (CHC; Schneider \& McGrew, 2012).

3 La cinquième version du WISC (Wechsler, 2016) confirme cette inscription théorique majeure, avec la volonté tout attendue de réactualisation des items pour une meilleure proximité avec le contexte de vie et les préoccupations quotidiennes de la personne évaluée, et des étalonnages de référence pour tenir compte de l'effet Flynn (Nisbett et al., 2012 ; Flynn, 2007 ; Grégoire, 2006 ; Wicherts et al., 2004). 
Ces évolutions majeures respectent l'approche de l'intelligence de Wechsler, en proposant des épreuves composites variées: un éventail le plus large possible de situations différentes est proposé à la personne, laissant place pour le.la psychologue à l'observation clinique.

5 Dans un premier temps, nous rappellerons les limites de la quatrième version du WISC et ce qu'apporte la dernière-née; puis nous exposerons les grandes lignes de la démarche d'interprétation en mettant l'accent sur l'analyse des épreuves composant l'échelle.

\section{De la version IV et la version $V$ de l'échelle de Wechsler pour enfants et adolescent.e.s}

\section{Limites de la version IV}

6 Avec la parution du WISC-IV, une architecture nouvelle de l'échelle fait son entrée : alors que le WISC-III (Wechsler, 1996) distingue de manière empirique une échelle verbale et une échelle de performance, le WISC-IV propose une organisation autour d'indices, représentant les grands facteurs du modèle hiérarchique de Carroll (1993).

7 Le passage de la version III à la version IV visait un rééquilibrage de la représentation de ces facteurs de groupe : en effet, dans le WISC-III, l'intelligence fluide, le traitement auditif, la vitesse de traitement et la mémoire avaient un rôle limité. Par contre, l'intelligence cristallisée et le facteur visuo-spatial occupaient une place plus importante.

8 Le transfert, dans la quatrième version, des subtests Information et Arithmétique en épreuves facultatives, l'introduction de nouvelles tâches, Identification des concepts, Barrage, Raisonnement verbal avait permis ce rééquilibrage des facteurs correspondants aux indices, avec une meilleure représentation de la mémoire de travail (Gwm) et de l'intelligence fluide (Gf), une diminution de l'impact de l'intelligence cristallisée (Gc).

9 Quatre indices avaient été identifiés dans le WISC-IV : compréhension verbale (ICV), raisonnement perceptif (IRP), mémoire de travail (IMT) et vitesse de traitement (IVT), alors que la troisième version ne disposait que de trois indices, qui plus est assez peu représentatifs des facteurs de groupe du fait d'un nombre de subtests insuffisant ou des subtests ne saturant pas suffisamment les différents facteurs.

10 Pour autant, cette quatrième version de l'échelle présentait des limites et des insuffisances :

- une représentation inégale des facteurs de groupes : les indices ICV et IRP étaient constitués par trois épreuves, alors que l'IMT et l'IVT n'en comportaient que deux; le modèle CHC n'introduisant pas de prédominance entre les différents facteurs de groupe, chaque indice devrait donc être appréhendé avec un nombre équivalent de subtests ;

- un nombre d'indices insuffisant au regard du nombre de facteurs de groupe du modèle $\mathrm{CHC}$; quatre indices seulement pour seize facteurs degroupe différents sur le niveau intermédiaire du modèle CHC (Schneider \& McGrew, 2012);

- un IRP trop hétérogène : il se composait d'épreuves relevant à la fois du facteur visuo-spatial (Gv) telles que Cubes et de l'intelligence fluide (Gf) comme Matrices. Pour résoudre ces subtests, une large gamme de compétences cognitives sont sollicitées: flexibilité mentale, 
catégorisation, capacité d'analyse, de manipulation mentale des formes spatiales au travers des différents subtests. Des résultats hétérogènes à cet indice ne permettent pas d'inférer des hypothèses quant au fonctionnement cognitif ; les subtests étant, de par leur nature et leur conception, trop disparates ;

- un IMT exclusivement centré sur des stimuli auditifs verbaux, occultant l'analyse visuelle et le traitement des configurations spatiales;

- un IVT ne mettant pas suffisamment en valeur le geste graphique mobilisé dans l'apprentissage de l'écriture pour le subtest code, et proposant une démarcation insuffisante entre les items stimuli et les items réponse pour le subtest Symbole ;

- le subtest Matrices se composant d'items hétérogènes, mêlant des traitements de nature différente et pas toujours simples à différencier (raisonnement analogique, raisonnement sur série, raisonnement inductif / déductif, multiplicité des opérations sollicitées, diversité des critères à croiser), renforçant ainsi la difficulté d'analyse des erreurs ;

- un outil de plus de dix ans, d'où la nécessité de mise à jour des items pour recontextualiser les attendus et assurer une meilleure correspondance entre les sollicitations du test et les préoccupations de la population d'une part ; et la nécessité de renouveler les étalonnages au regard des compétences des différentes générations d'autre part.

11 Par conséquent, les indices produits par le WISC-IV étaient de qualité inégale et pas toujours représentatifs des facteurs de groupe du modèle $\mathrm{CHC}$; les subtests n'étaient pas toujours suffisamment représentatifs de l'indice correspondant; certains items composant les subtests étaient obsolètes, de natures différentes, ou ne répondaient pas aux attendus, rendant ainsi le subtest hétérogène.

12 Ainsi, la nécessité de revoir l'ensemble des items au sein des indices s'imposait; de même que la nécessité de rééquilibrer la composition des indices et d'accroître le nombre d'indices structurant l'échelle pour une meilleure proximité avec le modèle CHC.

\section{Les améliorations apportées par la cinquième version}

La cinquième version affirme une inscription plus marquée dans le modèle $\mathrm{CHC}$ et une prise en compte plus fondamentale des recherches sur l'intelligence, en neurosciences, et des modèles de la mémoire humaine.

L'évolution majeure est la dissociation de l'indice de raisonnement perceptif pour créer deux indices : un indice visuo-spatial (IVS) formé des épreuves Cubes et Puzzles visuels (subtest repris de la WAIS-IV ; Wechsler, 2011) ; et un indice raisonnement fluide (IRF) comprenant les subtests Matrices et Balances (subtests présents également dans la WAIS-IV).

15 L'architecture de l'outil permet donc le passage de quatre indices à cinq indices principaux : indice de compréhension verbale (ICV), indice visuo-spatial (IVS), indice de raisonnement fluide (IRF), indice de mémoire de travail (IMT), indice de vitesse de traitement (IVT). Ces indices sont à rapprocher respectivement les facteurs de groupe intelligence cristallisée (Gc), traitement visuel (Gv), raisonnement fluide (Gf), mémoire à court terme (Gsm) et vitesse de traitement (Gs) du modèle Cattell / Horn et Carroll (CHC). Ainsi, cinq facteurs de groupe sur les seize actuels de ce modèle sont représentés. Plusieurs facteurs restent non couverts. Il manque notamment les processus auditifs $(\mathrm{Ga})$ qui peuvent être en partie approchés par l'épreuve facultative Séquence lettres-chiffres, les connaissances quantitatives $(\mathrm{Gq})$, à rapprocher de l'indice 
raisonnement quantitatif; la lecture et l'écriture (Grw) ou la mémoire à long terme (Glr) qui peut être sondée en analysant les épreuves de Vocabulaire et d'Information.

Des indices complémentaires peuvent également être calculés: l'indice de raisonnement quantitatif (IRQ) qui est un facteur spécifique de niveau I du raisonnement fluide dans le modèle $\mathrm{CHC}$ et l'indice de mémoire de travail auditive (IMTA), correspondant à l'IMT du WISC-IV.

Par conséquent, plusieurs facteurs de groupe du modèle $\mathrm{CHC}$ sont appréhendés dans cette nouvelle échelle.

18 Chaque indice se compose de deux épreuves, d'où un rééquilibrage de la représentation des différents facteurs de groupe au niveau des subtests, en cohérence avec le modèle $\mathrm{CHC}$.

19 Les qualités métriques sont améliorées ; l'outil s'adapte davantage aux potentialités et à l'implication de l'enfant: les items d'apprentissage sont plus nombreux, les consignes plus précises, des possibilités de reformulation et de relance pour un apport qualitatif plus importantes. Une volonté plus nette de structurer et d'accompagner l'apprentissage apparaît: le sujet peut observer, imiter, reformuler. L'introduction d'une note réponses partielles à Cubes permet la prise en compte de réalisations partielles par le sujet et introduit une plus grande progressivité dans la cotation.

Des règles d'arrêt plus rapides et un visuel mieux structuré à Symboles rendent la passation plus fluide et plus conviviale.

21 Tous les subtests ont été revus avec le changement d'items ou l'ajout de nouvelles modalités, comme, par exemple, l'introduction d'une troisième phase ordre croissant à Mémoire des chiffres pour renforcer l'évaluation de la mémoire de travail. Les signes de Code ont été renouvelés avec une exigence graphique plus importante (réalisation d'une boucle, tracé dissymétrique). La distinction des processus simultanés et des processus séquentiels par des items différents dans l'épreuve de Matrices apporte nuance et clarification dans l'appréhension des modalités de raisonnement du sujet. En effet, les items sont désormais de deux types seulement: en série (les formes géométriques sont présentées en ligne) ou sous forme d'analogies (les figures géométriques sont présentées sous forme de carré à quatre ou neuf cases). Les items en série nécessitent un traitement séquentiel alors que les items analogiques requièrent un traitement simultané (Kaufman, Raiford, \& Coalson, 2016).

22 Trois nouveaux subtests apparaissent: Puzzles visuels, Balances et Mémoire des images. Cette dernière épreuve permet ainsi d'introduire la dimension visuelle dans l'évaluation de la mémoire de travail.

23 Enfin, on note une extension des groupes cliniques: haut potentiel, handicap intellectuel, troubles des apprentissages, trouble de l'attention avec ou sans hyperactivité (TDA-H), autisme, traumatisme cérébral (données américaines essentiellement).

\section{Éléments d'interprétation}

24 Nous devons, dans l'espace qui nous est imparti, donner les grandes lignes de la démarche d'interprétation et non de rentrer dans le détail. L'analyse par le.la psychologue des résultats doit être à la fois rigoureuse et souple, ce qui peut paraitre de prime abord contradictoire. Rigoureuse, car il est nécessaire de s'appuyer sur les 
calculs statistiques pour pouvoir interpréter les résultats, identifier les données qui caractérisent de manière significative le profil du sujet, mais tout en gardant une certaine souplesse d'interprétation. L'objectif est de réaliser une interprétation raisonnée des résultats, mais que nous ne devons pas utiliser de manière mécanique et automatisée l'ensemble des indicateurs à disposition. C'est bien là l'apport du.de la psychologue que de croiser des données chiffrées avec son observation clinique, son (ses) cadre(s) théorique(s) de référence, les éléments issus des différents entretiens.

Comme pour les versions précédentes, la démarche se veut descendante : du général au particulier. Dans un premier temps, il s'agit de choisir, d'analyser et d'interpréter un ou plusieurs score(s) global(aux) parmi les quatre possibles : le quotient intellectuel total (QIT) qui repose désormais sur sept et non dix épreuves; l'indice d'aptitude générale (IAG) pouvant être utilisé lorsque le QIT est hétérogène, calculé à partir de toutes les épreuves de l'ICV, de l'IRF auxquelles s'ajoutent les Cubes; l'indice de compétence cognitive (ICC) calculé à partir des épreuves d'IMT et d'IVT ; et l'indice non verbal (INV) qui se compose de toutes les épreuves ne nécessitant pas de réponse verbale de la part de la personne évaluée.

Le choix dépend des objectifs visés par l'évaluation, des caractéristiques de la personne (pour un.e adolescent.e ayant des difficultés d'expression orale, il sera intéressant de comparer le QIT à l'INV), et des résultats obtenus.

Si ces scores sont jugés homogènes, on peut les caractériser en déterminant l'intervalle de confiance ${ }^{1}$ autour de ces derniers, le rang percentile ${ }^{2}$, la zone dite "d'efficience " allant de « très faible » à " très élevée » et en proposer une interprétation.

Il s'agit ensuite de s'intéresser aux indices de second ordre : les cinq indices principaux que sont l'ICV, l'IVS, l'IRF, l'IMT et l'IVT et les deux indices complémentaires, à savoir l'IRQ et l'IMTA si les subtests complémentaires ont été administrés (Arithmétique et Séquence lettre-chiffres). Il convient de savoir si chaque indice est homogène et donc représentatif de l'échelle, ou bien si la variabilité des notes standard au sein de l'indice est inhabituellement importante (différence significative et rare). Dans ce cas, il ne peut être résumé que par une note unique. À cette étape, nous devons également dégager les points forts et les points faibles de la personne, à la fois au niveau intra- et interindividuel. Rappelons que l'analyse interindividuelle consiste à comparer la note du sujet à celle obtenue par le groupe du même âge (comparaison à la moyenne standard) et l'analyse intra-individuelle, à repérer les forces et les faiblesses du sujet par rapport à ses propres performances (moyenne des notes obtenues aux subtests par le sujet).

Dans un troisième temps, les résultats aux subtests sont analysés, là encore d'un point de vue intra- et interindividuel. Pour mener cette analyse, nous proposons, dans la continuité du travail réalisé par Laporte (2007), des tableaux analytiques et descriptifs des différents subtests.

\section{Analyse des subtests}

Les tableaux synthétisent l'ensemble des données pour chacun des subtests. La construction des tableaux reprend: 
31 1. les consignes et les éléments de situations relatives à chaque subtest à partir du manuel d'administration des ECPA (stimuli, matériaux, manipulation, situation d'apprentissage) ; l'intérieur de chaque épreuve, il est enfin possible de mener des analyses complémentaires qui restent optionnelles et laissées à l'appréciation du.de la psychologue.

41 Le manuel propose plusieurs analyses des notes additionnelles: calcul d'une note standard pour cubes sans bonifications et cubes partiels puis comparaison avec la note standard de Cubes ; Mémoire des chiffres en ordre direct, en ordre indirect, et en ordre croissant, puis comparaison des différences entre ces scores standardisés ; observation des empans (Mémoire des chiffres, Mémoire d'images et Séquence lettres-chiffres), etc.

Ainsi, cette nouvelle version permet une analyse fine du fonctionnement cognitif de la personne évaluée qui ne prendra sens qu'en confrontant les éléments issus du WISC avec ceux émanant d'autres sources (entretiens, observations, autres outils d'évaluation).

\section{Conclusion}

Nous avons tenté en quelques lignes de montrer les apports de cette nouvelle version et de donner des pistes pour analyser, interpréter les résultats obtenus par la personne évaluée. La nouvelle échelle nous offre l'opportunité de construire une alliance entre approche scientifique basée sur un référent théorique et une approche clinique basée sur l'analyse de la relation intersubjective psychologue / consultant.e. La diversité des épreuves nous donne aussi une ouverture plus marquée sur la restitution dynamique, à savoir des adaptations des modalités de passation d'épreuves, au-delà de l'approche standardisée. Cette possibilité permet alors d'intégrer de nouveaux éléments d'interprétation et de compréhension dans l'ensemble du processus d'évaluation tout en favorisant l'exploitation pyschopédagogique. Cette dimension reste encore à explorer et à exploiter pour les psychologues de l'Éducation nationale, nous incitant à développer nos compétences et notre sens de l'investigation pour une meilleure

L'orientation scolaire et professionnelle, 47/1 | 2018 
compréhension de chaque adolescent.e, dans son contexte de vie et d'apprentissage. Ces pistes de réflexion viennent asseoir notre statut et confortent notre place au sein des équipes éducatives tant dans les établissements qu'en centre d'information et d'orientation (CIO) ou au sein des réseaux d'aides spécialisées aux élèves en difficulté (RASED).

\section{BIBLIOGRAPHIE}

Carroll, J. B. (1993). Human cognitive abilities. A survey of factor-analytic studies, New York: Cambridge University Press.

Flynn, J. R. (2007). What is intelligence? Cambridge: Cambridge University Press.

Grégoire, J. (2006). Devenons-nous plus intelligents ? Le Journal des psychologues, 1, 38-42.

Kaufman, A. S., Raiford, S. E., \& Coalson, D. L. (2016). Intelligent testing with the WISC-V, Hoboken, New Jersey, Wiley.

Laporte, E. (2007). Analyse cognitive des tâches impliquées dans les épreuves du wisc4, L'Orientation scolaire et professionnelle, 36(3), 438-460.

Nisbett, R. E., Aronson, J., Blair, C., Dickens, W., Flynn, J., Halpern, D. F., \& Turkheimer, E. (2012). Intelligence: new findings and theoretical developments. American psychologist, 67(2), 130.

Schneider, W. J., \& McGrew, K. (2012). The Cattell-Horn-Carroll model of intelligence. In D. Flanagan \& P. Harrison (eds.), Contemporary Intellectual Assessment: Theories, Tests, and Issues ( ${ }^{r d}$ ed.) (pp. 99-144). New York: Guilford.

Wechsler, D. (1996). Manuel d'interprétation et de cotation du WISC-III. ECPA.

Wechsler, D. (2005). Manuel d'interprétation et de cotation du WISC-IV. ECPA.

Wechsler, D. (2011). Manuel d'interprétation et de cotation de la WAIS-IV. ECPA.

Wechsler, D. (2016). Manuel d'interprétation et de cotation du WISC-V. ECPA.

Wicherts, J. M., Dolan, C. V., Hessen, D. J., Oosterveld, P., Van Baal, G. C. M., Boomsma, D. I., \& Span, M. M. (2004). Are intelligence tests measurement invariant over time? Investigating the nature of the Flynn effect. Intelligence, 32(5), 509-537.

\section{NOTES}

1. . L'intervalle de confiance (IC) à $95 \%$ ou $90 \%$ selon le seuil choisi, est un intervalle de valeurs qui a $95 \%$ ou $90 \%$ de chance de contenir la vraie valeur du paramètre estimé.

2. . Le rang percentile indique la proportion de personnes qui ont un score égal ou inférieur à ce rang percentile. Par exemple, un rang percentile de 25 indique que $25 \%$ de la population en question a un score égal ou inférieur à celui de l'individu. 


\section{RÉSUMÉS}

Les psychologues de l'Éducation nationale sont de plus en plus sollicité.e.s par les familles, les équipes éducatives, les commissions et les jeunes eux-mêmes pour apporter leurs regard et expertise sur des problématiques variées (difficultés scolaires, réorientations, situations de handicap, etc.). Dans le cadre des diverses demandes dont il.elle.s peuvent être le.la dépositaire, certaines d'entre elles pourront nécessiter le recours à un bilan psychologique avec éventuellement l'utilisation d'une échelle de Wechsler comme le WISC.

L'objectif de cet article est de montrer en quoi les apports de la nouvelle version du WISC parue en octobre 2016 répondent en partie aux limites de la version précédente ; d'apporter quelques éléments sur l'interprétation des résultats en mettant l'accent sur l'analyse des quinze subtests qui composent l'échelle.

Psychologists working within the national education system are increasingly asked by families, educational teams, commissions and young people themselves to bring their expertise to bear on a range of problems (academic difficulties, change of direction, disability, etc). With regard to the range of requests they may receive, some may require the use of a psychological assessment, possibly using a Wechsler scale such as the WISC. The aim of this article is firstly to show how the new version of the WISC published in October 2016 responds, in part, to the limitations of the previous version, and secondly, to contribute to the interpretation of the results with an emphasis on analysing the fifteen subtests that make up the scale.

\section{INDEX}

Mots-clés : intelligence, WISC-V, analyse, bilan, interprétation

Keywords : Intelligence, WISC-V, assessment, interpretation

\section{AUTEURS}

\section{EVELYNE LAPORTE}

est Psychologue de l'Éducation nationale, Directrice de centre d'information et d'orientation, Saint-Malo. Contact : CIO de Saint-Malo, 26 Quai du Val, 35400 Saint-Malo. Courriel :

Evelyne.Laporte@ac-rennes.fr

\section{KATIA TERRIOT}

est Psychologue de l'Éducation nationale, Chargée d'enseignement et de recherche, Inetop Cnam, Paris. Thèmes de recherche : évaluation psychologique, intelligence, motivation, estime de soi. Courriel : katia.terriot@lecnam.net 